\title{
Experimental comparison of Fiber Bragg Grating installation techniques for aerospace systems
}

\author{
Pier Carlo Berri ${ }^{1}$, Matteo D.L. Dalla Vedova ${ }^{1, *}$, and Paolo Maggiore ${ }^{1}$ \\ ${ }^{1}$ Dept. of Mechanical \& Aerospace Engineering (DIMEAS), Politecnico di Torino, Turin, Italy
}

\begin{abstract}
Aircraft systems are becoming more and more complex, as they are required to perform multiple functions. For example, smart systems need to be able to self-monitor their working parameters, in order to infer their health status. All these additional functions require the system to acquire a multitude of measurements; albeit sometimes it is possible to implement virtual sensor techniques, dedicate sensing hardware is usually needed. As a main drawback, the installation of the needed sensors adds up to the total complexity, weight, cost and failure rate of the system. In this context, minimally invasive sensors can be used to measure the system parameters with high spatial resolution and minimal added complexity. One key technology in this field is the Fiber Bragg Gratings (FBG) optical sensors, used to perform strain and temperature measurements. This work describes an experimental campaign intended to assess and validate several installation techniques for FBGs as strain sensors. Two test benches were developed for different measurement setups. One is intended for creep and repeatability tests of a FBG sensor glued at both ends; the other was used to compare point gluing and continuous gluing techniques on an aluminium beam subject to a bending load. Results are compared with numerical simulations of the structure and measurements performed with traditional strain gages.
\end{abstract}

\section{Introduction}

Next generation equipment for aircraft systems will be required to perform multiple secondary functions alongside with their primary one. Among those, Prognostics and Health Monitoring (PHM) tasks are one of the most important features that can enable a lifecycle cost reduction and an increase in availability of the system [1-5].

Usually, the PHM process for a system can be split into three phases [6]. At first, some quantities sensitive to faults are measured during the system operation. Then, data is analysed in order to detect and identify the early signs of faults, in the so-called Fault Detection and Identification (FDI) phase. Eventually, the information about the system health status is employed to estimate its Remaining Useful Life RUL.

\footnotetext{
${ }^{*}$ Corresponding author: matteo.dallavedova@polito.it
} 
The FDI phase needs to estimate the health status with very high precision, since a small error in FDI can lead to a large RUL estimation uncertainty. Then, the system selfmonitoring routines are required to acquire as much information as possible, with very high accuracy. The sensors already present for control purposes can sometimes be employed, but often a successful PHM requires more information [7-11]. However, the addition of sensing hardware has the downside of increasing costs and weight, as well as reducing the basic reliability of the system. In this context, new technologies can help providing sensors that combine high accuracy, small form factor and high reliability.

\subsection{Fiber Bragg Grating sensors: operation and applications}

In this work we focus on a particular class of optical sensors called the Fiber Bragg Gratings (FBGs) [12-16]. They consist in an optical fiber equipped with sensitive spots, able to detect variations of mechanical strain and temperature with very high precision.

An optical fiber is a thin glass filament composed of different layers. Starting from the outside, the coating is a protective polymer layer, typically at least 250 microns in diameter, required to handle the fiber and avoid accidental damages during manufacturing and installation. The cladding is a first glass layer (usually 125 microns in diameter), needed to direct and guide the light beam inside the fiber. The inner layer of the fiber is called the core, which has a higher refraction index than the cladding. The typical core diameter for a single-mode fiber is 8 microns. In fact, light propagates inside the core and is unable to escape through the cladding because it is confined by total internal reflection $[12,17]$ at the core-cladding interface. This way, it is possible to make a light beam follow a curved path inside the fiber.

The sensitive part of the sensor is the Fiber Bragg Grating. It consists in a small region, some millimeters long, where the refraction index of the core is periodically modulated.

During the operation of the sensor, a light beam with variable wavelength is sent into the fiber. The FBG acts like a selective mirror: most light is transmitted through the fiber and exits at the other end, while a selected wavelength $\lambda_{B}$ is reflected. This wavelength is dependent on the refraction index of the fiber $n$ and the spacing of the grating $\Lambda_{G}$ :

$$
\lambda_{B}=2 n \Lambda_{G}
$$

When the fiber is subject to a variation in mechanical strain $\Delta \varepsilon$, the FBG spacing changes and so the FBG wavelength; similarly, a temperature change $\Delta T$ causes thermal expansion and variation of the refraction index, also changing the wavelength:

$$
\Delta \lambda_{B}=K_{\varepsilon} \Delta \varepsilon+K_{T} \Delta T
$$

where $K_{\varepsilon}$ and $K_{T}$ are the characteristic coefficients of the fiber. FBGs are a particularly promising technology, since they provide minimally invasive measurements, thanks to their small dimensions, combined with very high accuracy and sensitivity. Several sensors can be stacked along the same optical fiber, provided they have a different nominal wavelength, and the fiber itself can be used for data transmission at the same time. This way, FBGs enable to build a sensing network characterized by light weight, high robustness, reliability and redundancy, small size, and relatively low cost.

Possible measurements of interest for system prognostics include structural health monitoring, vibration, pressure and temperature of hydraulic fluid, thermal monitoring of electrical or mechanical equipment, as well as the load determination of flight control actuators.

Strain measurements performed with FBGs can achieve accuracy in the order of a single microstrain or lower. As regards thermal measurements, FBGs provide an almost 
instantaneous response due to their small thermal capacity [18-21]. Additionally, this technology is robust to harsh environment and totally insensitive to EM disturbances, since all the sensing and signal transmission is performed optically, and the materials employed in FBG manufacturing (mainly glass) are chemically inert.

\subsection{Aim of this work}

The main downside of FBGs is their lack of mechanical strength. They are very easily damaged if handled improperly, especially during installation and manufacturing. Moreover, a proper installation is a critical factor to achieve precise measurements: for example, the connection of the sensor to the monitored structure shall transmit loads and displacements with very high stiffness, minimizing the elastic deformation of the glue layer [22]. In this work we describe two experiments performed at Politecnico di Torino, intended to assess the effectiveness of different installations for strain measurements.

\section{First experiment: installation for strain measurements}

In the first experiment, we compare different gluing techniques for installing the fiber on the structure [23]. Specifically, we consider a continuous gluing of an FBG equipped fiber onto a structural beam, as well as a point gluing aiming to achieve a uniform strain in the whole FBG. The measures are validated through comparison with a finite element simulation performed with Nastran-Patran and with the readings of traditional resistive strain gages, installed near the FBGs. The simple structure considered would have allowed for a simpler numerical solution using the Euler-Bernoulli beam theory; however, the authors preferred a 2D finite element solution to account for local deformations (e.g. due to the interaction with the support structure or the load actuator), which may affect the measurements.

\subsection{Setup}

The block diagram of Figure 1 represents the experimental setup. The test sample is either an aluminum beam or a composite spar, clamped at one end, and loaded by imposing a displacement with a micrometric linear actuator at the other end. A fiber equipped with three FBGs is glued to the beam, and provides strain measurements. Each FBG is sided by two traditional strain gages to validate the measurements.

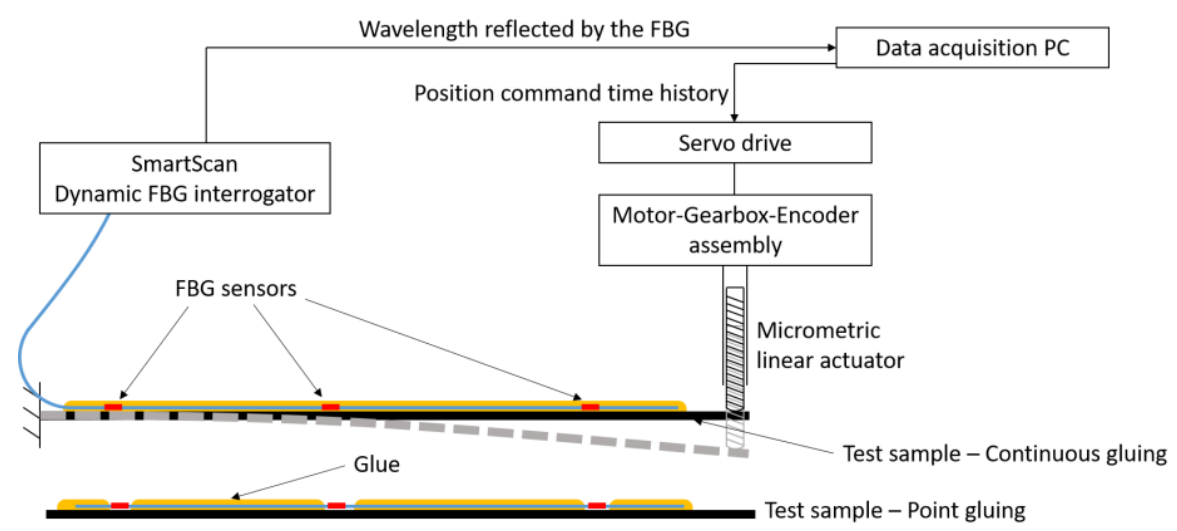

Fig. 1. Setup of the first experiment. 
Several tests have been performed, comparing different gluing techniques. The method providing the best compromise between flexibility and control over the thickness of the glue layer consisted in applying pressure to the glue through a soft layer. Additionally, we compared the performances of a continuous gluing and a point gluing (Figure 1). Continuous gluing provides better protection to the fiber, since it is completely embedded in the glue layer, while point gluing aims at generating a uniform strain in the whole FBG length, to minimize the wavelength dispersion.

\subsection{Results and discussion}

The left graph in Figure 2 shows the result of one of the measurements in terms of strain versus displacement. The red dashed curve is obtained through a Nastran-Patran finite element simulation, while the green lines indicate an acceptable range of the measurement. In fact, being the glue layer thickness not negligible with respect to the total thickness of the beam, the sensor cannot be assumed to be placed exactly on the beam surface. The green curves correspond, through the Navier equation, to the strain experienced by a sensor placed exactly on the beam surface or floating on the glue layer, as shown in the cross section of Figure 2 (detail in the left graph).

In this experiment we compared punctual and continuous gluing of the fiber, obtaining basically the same performance. In fact, a slightly higher dispersion is found for the continuous gluing, since the strain is not constant along the whole FBG, but this behavior was observed to have a negligible effect on the measurement accuracy.

At the right of Figure 2, the FBG measurement is compared to the average of two strain gages placed aside the fiber. The FBG slightly overestimates the strain, probably being its glue slightly thicker, but its measurement is much more immune to signal noise and requires less filtering. As a matter of fact, when the test sample is kept with a steady deformation, the FBG measure is almost stationary, while the strain gages need more signal conditioning being affected by electromagnetic noise.

At the end of this experimental campaign, we concluded that the main uncertainty for this setup is due to the unknown thickness of the glue layer, which is hard to control during installation, especially on complexly shaped structures. However, the installation on a real structural component will be less sensitive to this error since the structure characteristic dimensions will be orders of magnitude greater than the glue.

We concluded that the obtained results lie inside the expected interval, and the continuous vs point gluing have basically the same behavior. Then, continuous gluing is to be preferred in field applications, since it provides higher robustness and protection to the fiber, without significantly affecting the measures.
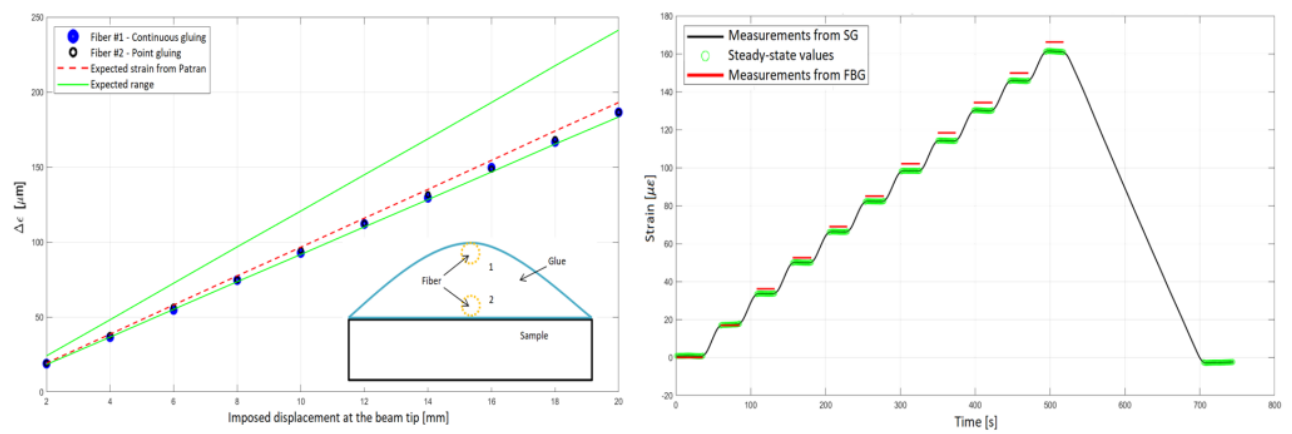

Fig. 2. Comparison of FBG and FEM (left) and validation through traditional strain-gages (right) 


\section{Second experiment: repeatability, aging and robustness}

A second experiment was meant to assess the long-term repeatability of the measures, evaluating phenomena as thermal dilation, as well as hygroscopic behaviour and viscouselastic response of the glue [23, 24]. As FBGs are sensitive to temperature variations, this experiment evaluated, among other aspects, whether a complete thermal compensation is required for measurements in a semi-controlled environment.

\subsection{Setup}

To avoid the glue height uncertainty of the previous experiment, we chose a setup allowing to apply directly an axial displacement to the fiber, without gluing it on a deformable structural component. As shown in Figure 3, the fiber is stretched between a micrometric linear actuator and a fixed support. The bonding between the support plates and the fiber is achieved by means of different glues, specifically three epoxy resins and a cyanoacrylate glue. The rest of the architecture is the same of the previous experimental setup, but here the imposed is easily computed knowing the end displacement and the initial free length $l_{0}$ of the fiber: then no FEM simulations or strain gages are needed.

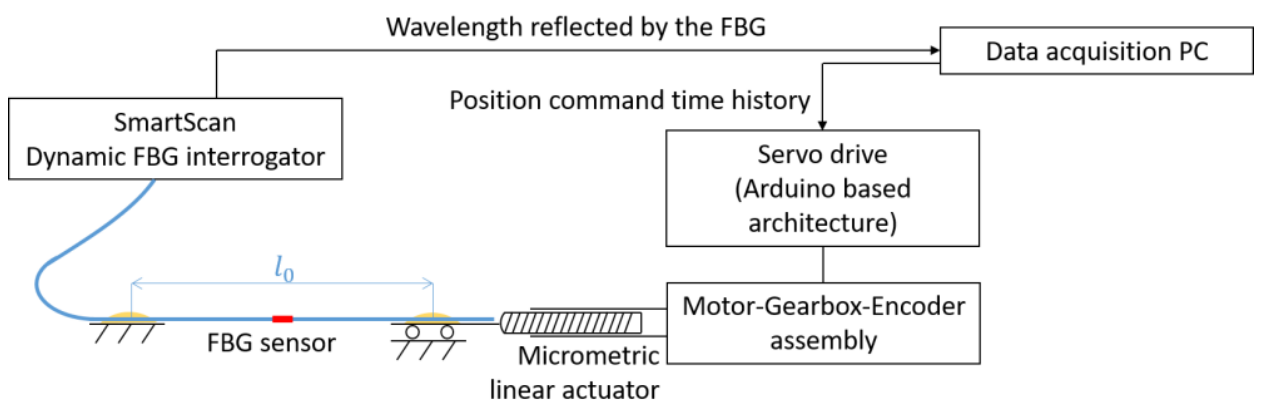

Fig. 3. Setup of the second experiment.

\subsection{Results and discussion}

Applying several times a small amplitude step command to the micrometric actuator, we observe a viscous elastic response due to the glue (Figure 4). The measure decays to about $98 \%$ of the step amplitude with a characteristic time in the order of one minute. This deformation is recovered during the descending step, and the behavior is repeatable. The noise affecting the signal, higher than the previous experiment, is actually due to the fiber capturing acoustical noise and air circulation through the setup, and not to the sensor itself.

The test was prolonged for several days, in order to evaluate unrecovered creep of the glue, as well as the effect of temperature and environmental humidity with different glues. Figure 5 shows the data acquired over a month period regarding room temperature and strain variation with respect to the initial acquisition, for four different fibers. Each FBG is bonded with a different commercial glue. The room temperature is actively controlled by the HVAC system of the facility, although with variations of some kelvins. A significant correlation between strain and temperature is observed. 

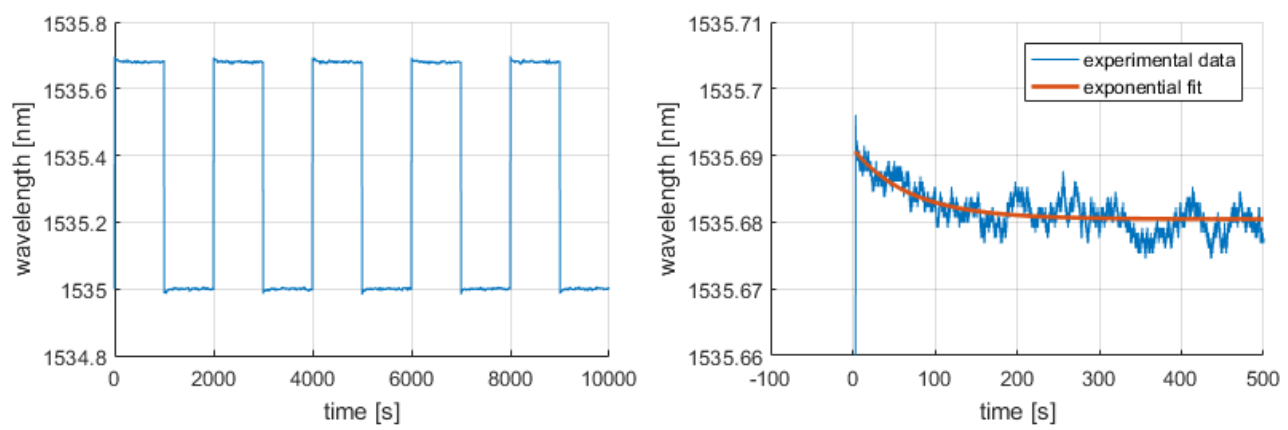

Fig. 4. Repeatability test highlighting the viscous-elastic behaviour of the glue (left); detail of the glue settling and exponential fit (right).

By linearly fitting the wavelength as a function of temperature, we find a slope compatible with the thermal dilation coefficient of the aluminum breadboard on which the setup is assembled. In the considered experimental setup, the thermal dilation of the fiber itself has no effect on the FBG, since it is completely compensated by the displacement imposed by the supporting structure; the effect of variation of the refraction index, although present, is smaller than the thermal expansion of the breadboard.
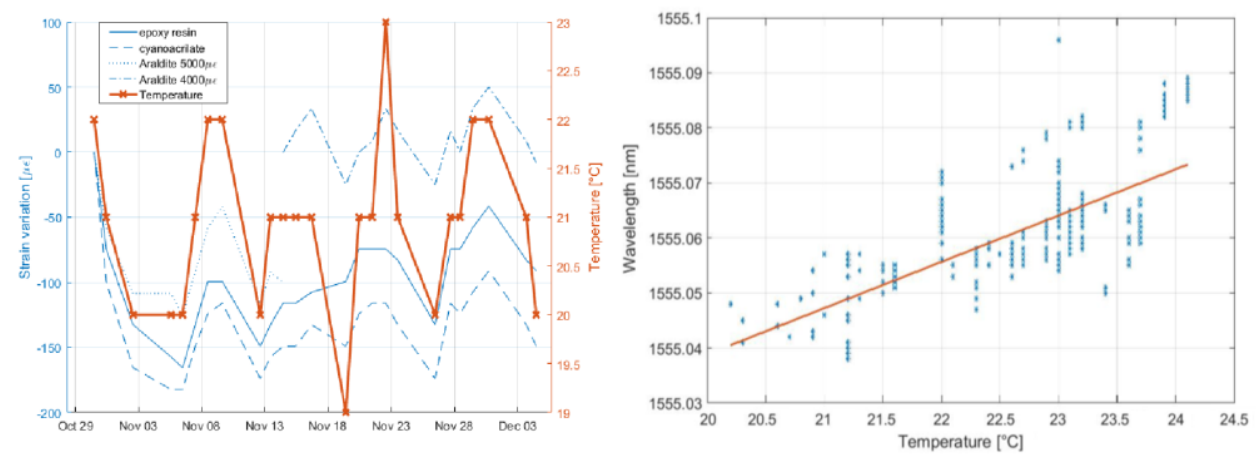

Fig. 5. Variation of measured strain and room temperature versus time (left); Linear fit of wavelength versus room temperature (right).

The linear dependency of strain from temperature allows to compensate the thermal effects by subtracting the variation due to temperature. The temperature compensated strain signal is shown in Figure 6: a linear dependency upon ambient humidity is found, and a fitting curve can be computed. This sensitivity to humidity is likely caused by a deformation of the glue, which is the only hygroscopic component of the setup. However, this behavior is difficult to model analytically.

By compensating also the humidity effect, we obtain the data points shown in Figure 7. The compensated strain is steadier than the original raw signal, meaning that the main disturbances have been taken into account. Additionally, a slow viscous elastic creep can be observed, with a settling time of about one week. 

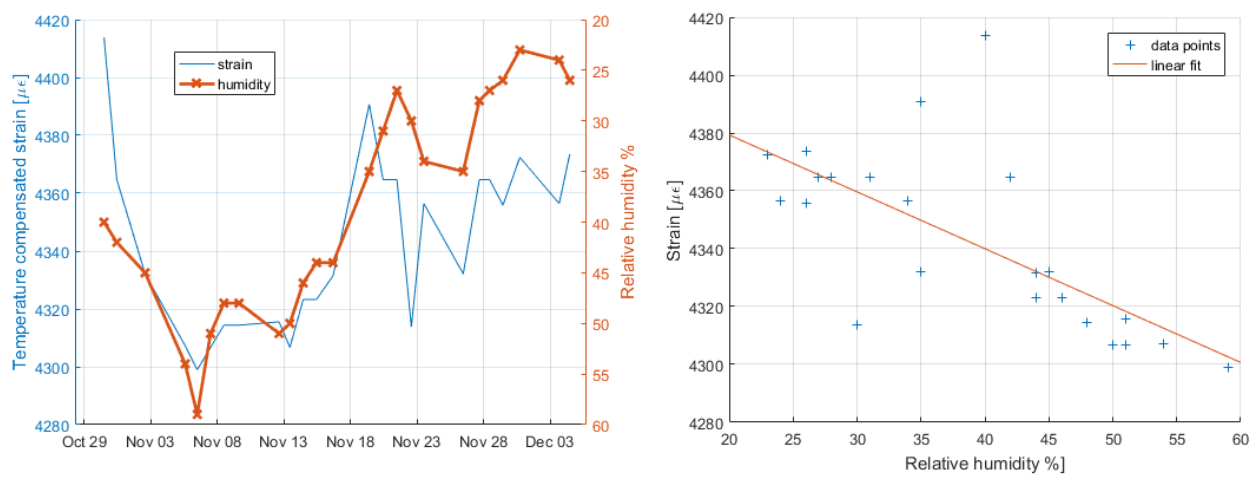

Fig. 6. Temperature compensated strain and humidity (left); linear fit of strain vs humidity (right); example for the Epoxy Resin sample.

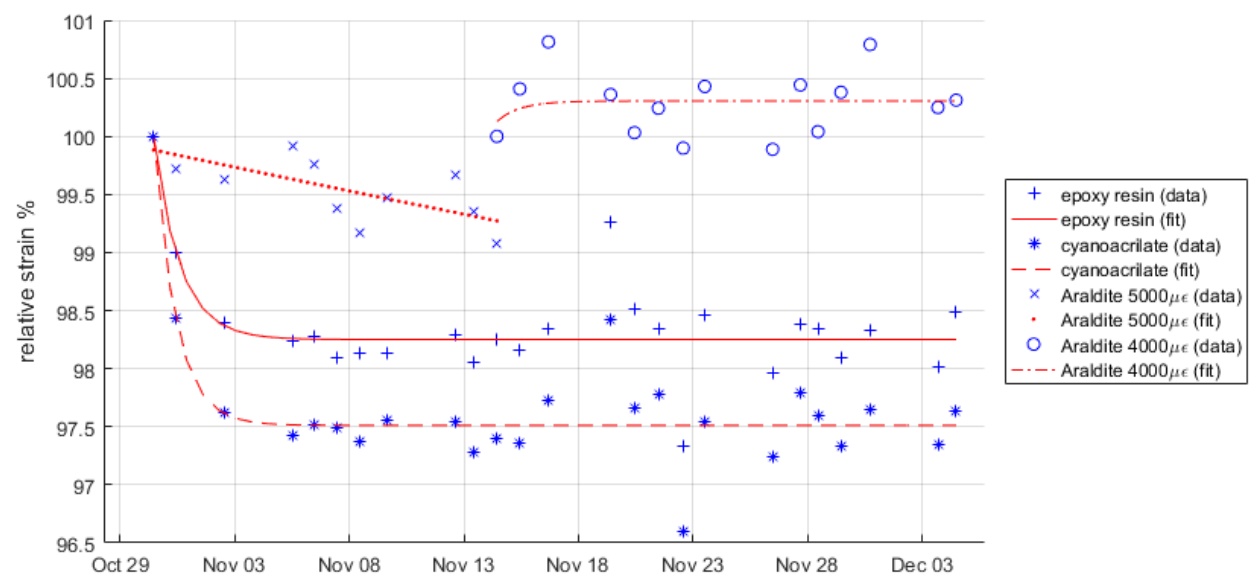

Fig. 7. Relative compensated strain (as a fraction of initial value) and exponential fit. Note that the two Araldite samples have too few data points for an effective fitting

We concluded that the most relevant measurement error was due to the ambient temperature fluctuation, due to the thermal expansion of the whole setup, the other effects being less significant. Other measurements in a more controlled environment will be required, in order to gather more data and assess the variance due to the manufacturing process.

\section{Conclusions and Scope}

Two experimental campaigns have been performed to assess and compare different installation techniques for FBG sensors. The results highlighted that the considered technology allows for high accuracy measures with minimally invasive installations, while the sensitivity of the strain measures to environmental effects was assessed.

Additional data will be gathered to determine the uncertainty related to manufacturing and installation tolerances, as well as to measure the glue creep at different temperature ranges. Moreover, structural strain measurements will be performed in flight on a small UAV platform with composite structure, and the results will be correlated to the actuator load via data fusion techniques. 
The authors wish to thank the Photonext Interdepartmental Center at Politecnico di Torino and the LINKS laboratory for their financial and technical support.

\section{References}

1. O. Benedettini, T.S. Baines, H.W. Lightfoot, R.M. Greenough, Inst. of Mechanical Engineers, Part G: J. of Aerospace Engineering, 223(2) 157-170 (2009)

2. Z. Williams, IEEE Aerospace Conference 9 (2006)

3. J. Yan, Machinery Prognostics and Prognosis Oriented Maintenance Management 183-239 (John Wiley \& Sons 2014)

4. G. Vachtsevanos, F.L. Lewis, M. Roemer, A. Hess, B. Wu, Intelligent Fault Diagnosis and Prognosis for Engineering Systems (John Wiley \& Sons, 2008)

5. A. De Martin, S. Autin, J. Socheleau, A. Dellacasa, G. Jacazio, G. Vachtsevanos, Annual conference of the Prognostics and Health Management Society (2018)

6. P.C. Berri, M.D.L. Dalla Vedova, L. Mainini, AIAA Scitech Forum (AIAA Paper 20192210) (2019)

7. R.A. Collacott, Mechanical Fault Diagnosis and condition monitoring 41-75 (Springer, Dordrecht 1977)

8. C. Commault, J.M. Dion, S.Y. Agha, 4th Workshop on Advanced Control and Diagnosis (2006)

9. L. Mainini, K.E. Willcox, 18th AIAA/ISSMO Multidisciplinary Analysis and Optimization Conference (2017)

10. L. Mainini, K.E. Willcox, Computers \& Structures 182 296-312 (2017)

11. A. Suarez, G. Heredia, A. Ollero, J. of Sensors 4515828 (2018)

12. J.L. Santos, F. Farahi, Handbook of Optical Sensors (CRC Press, 2018)

13. S.J. Mihailov, MDPI Sensors 12(2) 1898-1918 (2012)

14. S.J. Mihailov, D. Grobnic, C. Hnatovsky, R.B. Walker, P. Lu, D. Coulas, H. Ding MDPI Sensors 17(12) 2909 (2017)

15. J. Habel, T. Boilard, J.S. Frenière, F. Trépanier, M. Bernier, MDPI Sensors 17(11) 2519 (2017)

16. D. Grobnic, S. Mihailov, C.W. Smelser, H. Ding, IEEE Photonics Technology Letters 16(11) 2505-2507 (2004)

17. D. Ahuja, D. Parande, J. of Scientific Research and Reviews 1(5) 060-068 (2012)

18. N. Hirayama, Y. Sano, ISA Transactions 39(2) 169-173 (2000)

19. J. Jung, H. Nam, B. Lee, J.O. Byun, N.S. Kim, Applied Optics 38(13) 2752-2754 (1999)

20. W. Chen, R. Gassino, Y. Liu, A. Carullo, G. Perrone, A. Vallan, D. Tosi, Proc. IEEE Int. Symposium on Medical Measurements and Applications (MeMeA 2015)

21. D. Zhang, J. Wang, Y. Wang, X. Dai, Measurement Science and Technology 25(7) (2014)

22. P.C. Berri, M.D.L. Dalla Vedova, P. Maggiore, T. Scolpito, Journal of Physics: Conference Series 1249012015 (2019)

23. Y. Zhang, L. Zhu, F. Luo, M. Dong, R. Yang, W. He, X. Louet, IEEE Sensors Journal, 16(15) 5958-5963 (2016).

24. N. Tanaka, Y. Okabe, N. Takeda, Smart Mat. \& Struct. 12(6) 940-946 (2003) 